\title{
O USO DE VESTÍGIOS E DE ARMADILHAS FOTOGRÁFICAS COMO FERRAMENTA PARA A COMPLEMENTAÇÃO DE INVENTÁRIOS DE MAMÍFEROS NA SERRA DA JIBOIA, RECÔNCAVO BAIANO Bianca de Moura Calixto ${ }^{1}$; Téo Veiga de Oliveira; \\ 1. Bolsista PROBIC/UEFS, Graduando Ciências Biológicas, Universidade Estadual de Feira de Santana, e-mail: ban_calixtof2@hotmail.com \\ 2. Orientador, Departamento de Ciências Biológicas, Universidade Estadual de Feira de Santana, e-mail: teovoli@yahoo.com.br
}

PALAVRAS-CHAVE: pegadas; armadilha fotográfica; mamíferos.

\section{INTRODUÇÃO}

Mamíferos terrestres de médio e grande porte são fundamentais na manutenção do equilíbrio das florestas neotropicais. Estes animais desempenham um importante papel em vários níveis da organização de um ecossistema (Emmons \& Feer, 2005; Abreu Jr. \& Kohler, 2009; Sinclair, 2003; Tonhasca Jr., 2005); mamíferos maiores são importantes também por agruparem diversas espécies consideradas indicadoras ambientais, refletindo a preservação do local onde ocorrem (Mazzolli, 2006).

Apesar disto, mamíferos terrestres de médio e grande porte são pouco estudados devido às dificuldades referentes ao seu registro. Uma das formas mais seguras de aumentar a representatividade dos mamíferos médios e grandes nos inventários é registar estes animais não através de sua captura, mas a partir da recuperação dos vestígios deixados por eles em seu ambiente e de registros visuais diretos ou fotográficos. Além de se tratar de uma importante ferramenta no registro de espécies não registradas através dos demais métodos, o registro através de vestígios é uma metodologia fácil de ser desenvolvida e com baixo custo.

Além dos vestígios, a instalação de armadilhas fotográficas na área estudada também auxilia na elaboração de inventários mais completos, já que registra espécies comumente não capturadas e mesmo aquelas que ocorrem em locais onde os vestígios não são muito comuns. Esse trabalho objetivou, portanto, ampliar o uso de metodologias de registro indireto (vestígios) e diretos (armadilhas fotográficas) no inventário de mamíferos realizado na Serra da Jiboia, Bahia.

\section{METODOLOGIA}

As expedições de campo foram desenvolvidas na Serra da Jiboia, na região do Recôncavo Baiano, nos municípios de Varzedo, Elísio Medrado, Santa Terezinha e Castro Alves. Durante as caminhadas para o desenvolvimento das demais atividades ligadas ao projeto vinculado a este plano de trabalho foram procurados ativamente sinais de vestígios deixados por mamíferos de médio e grande porte em trilhas no interior da mata e em sedimentos próximos a córregos.

Diversos tipos de vestígios podem ser coletados, os mais típicos e identificáveis são as pegadas e trilhas. Estas pegadas, entretanto, podem ser registradas de forma mais definitiva do que as fotografias: com a confecção de moldes em gesso (Reis et al., 2010).

O processo de fabricação de moldes é muito simples: uma "fatia" de um cano de PVC de $100 \mathrm{~mm}$ de diâmetro é suavemente acomodada ao redor do vestígio para evitar que este seja deformado ou destruído; em seguida uma pasta de gesso é produzida, com uma consistência 
tal que possa preencher os detalhes da pegada e despejada no interior da "fatia" de PVC. Após seco, o molde deve ser retirado delicadamente do substrato e lavado para retirar o excesso de areia, lama e outras impurezas (Reis et al., 2010).

Os moldes limpos e secos foram identificados com o auxílio de guias de vestígios, como os de Becker \& Dalponte (1999) e Borges \& Tomás (2004). As pegadas foram tombadas na Seção de Icnologia da Coleção de Mamíferos da Divisão de Mamíferos do Museu de Zoologia da UEFS para servirem de referência a outros estudos.

Também foram instaladas armadilhas fotográficas em pontos da mata que sugeriam o trânsito de mamíferos. Próximo a cada armadilha foi amarrada a uma árvore uma isca odorífera e alguns atrativos foram jogados no chão, à frente das armadilhas, para atrair animais para a área de ação das câmeras. As armadilhas permaneceram ligadas ininterruptamente desde o início da campanha até o mês seguinte, quando eram trocadas de lugar (O’Connel et al., 2011; Glen et al., 2013).

\section{RESULTADOS E DISCUSSÃO}

Na Reserva Jequitibá foram fotografados Didelphis aurita, Nasua nasua (Fig. 1D), Procyon cancrivorus (Fig. 1A), Leopardus pardalis (Fig. 2), Cuniculus paca (Fig. 1B), Dasy procta sp. (Fig. 1C), Pecari tajacu, Dasypus novemcinctus (Fig. 1G) e Tamandua tetradactyla (Figs. 1F e 2), totalizando 53 registros (registros muito próximos no tempo foram contabilizados como sendo o mesmo). Nesta área destaca-se o registro da tentativa de predação de Leopardus pardalis sobre Tamandua tetradactyla, resultando em uma sequência de fotografias de quase trinta minutos (Fig. 2).

$\mathrm{Na}$ Fazenda Baixa de Areia, onde houve um total de 32 registros, foram fotografados Metachirus nudicaudatus (espécie de pequeno porte), Tamandua tetradactyla, Procyon cancrivorus, Nasua nasua, Cuniculus paca e Pecari tajacu (Fig 1H). Foram também encontradas pegadas de Pecari tajacu, (Fig. 3D) Procyon cancrivorus (Figs. 3B e 3C) e Dasypus novemcinctus (Fig. 3A). Já na RPPN Guarirú, com um total de 26 registros, foi registrado Nasua nasua (Fig. 1D), Cuniculus paca, Leopardus pardalis, Procyon cancrivorus e Eira barbara (Fig. 1E).

As regiões de Baixa Grande e Pioneira não tiveram resultados positivos. A Reserva Jequitibá foi o ponto com maior abundância de registros, refletindo a pouca interferência humana sobre o fragmento, enquanto que nas outras áreas a presença relatada de caçadores e a entrada de gado bovino em porções da mata podem ter influenciado negativamente na presença de mamíferos silvestres de maior porte. 


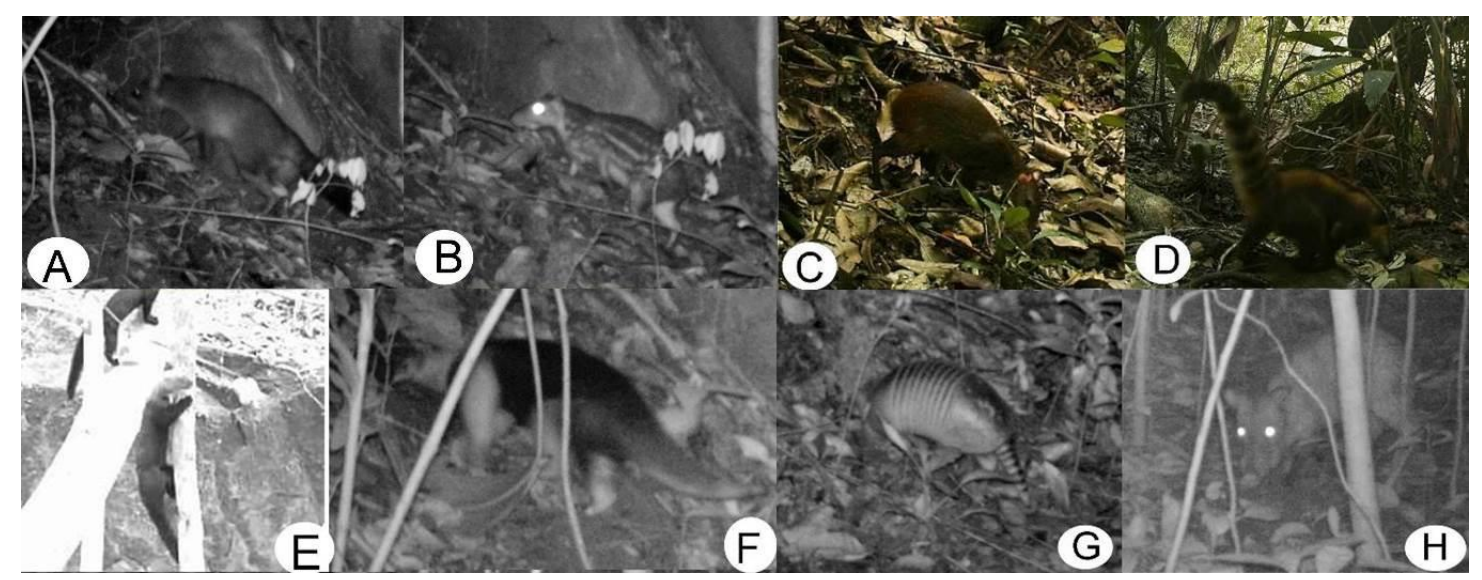

Figura 1: Animais fotografados na Serra da Jiboia. A: Mão-pelada (Procyon cancrivorus); B: Paca (Cuniculus paca); C: Cutia (Dasyprocta sp.); D: Quati (Nasua nasua); E: Irara (Eira barbara); F: Tamanduá-mirim (Tamandua tetradactyla); G: Tatu-verdadeiro (Dasypus novemcinctus) e H: Caititú (Pecari tajacu ).

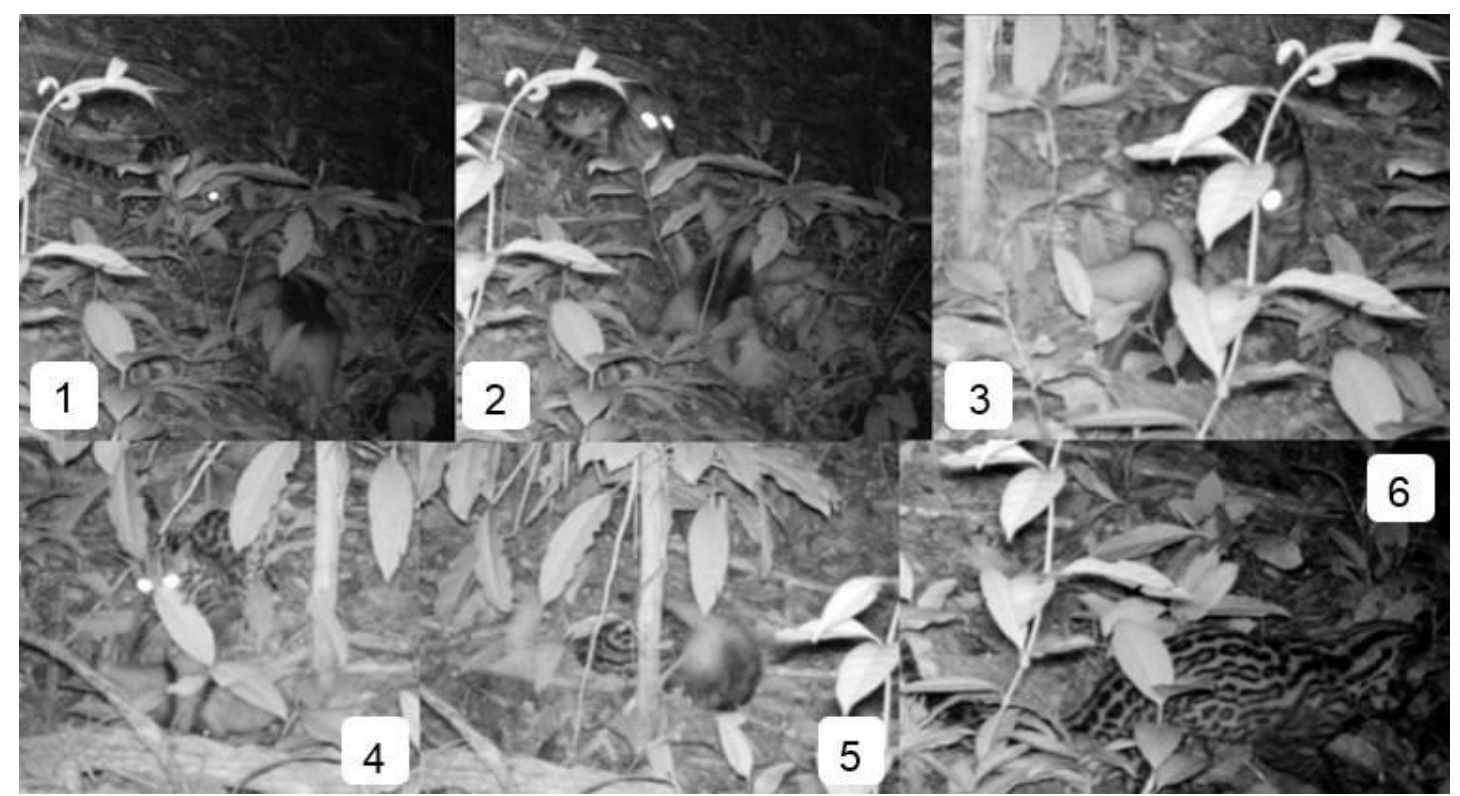

Figura 2: Sequência de quase 30 minutos da tentativa de predação de uma jaguatirica (Leopardus pardalis) sobre um tamanduá-mirim (Tamandua tetradactyla). Nas cenas 2,3 e 4 é possível ver o tamanduá em sua postura defensiva, com os braços abertos; na cena 5, os dois animais estão efetivamente lutando; na cena 6 a jaguatirica aparece sozinha no campo de ação da câmera.

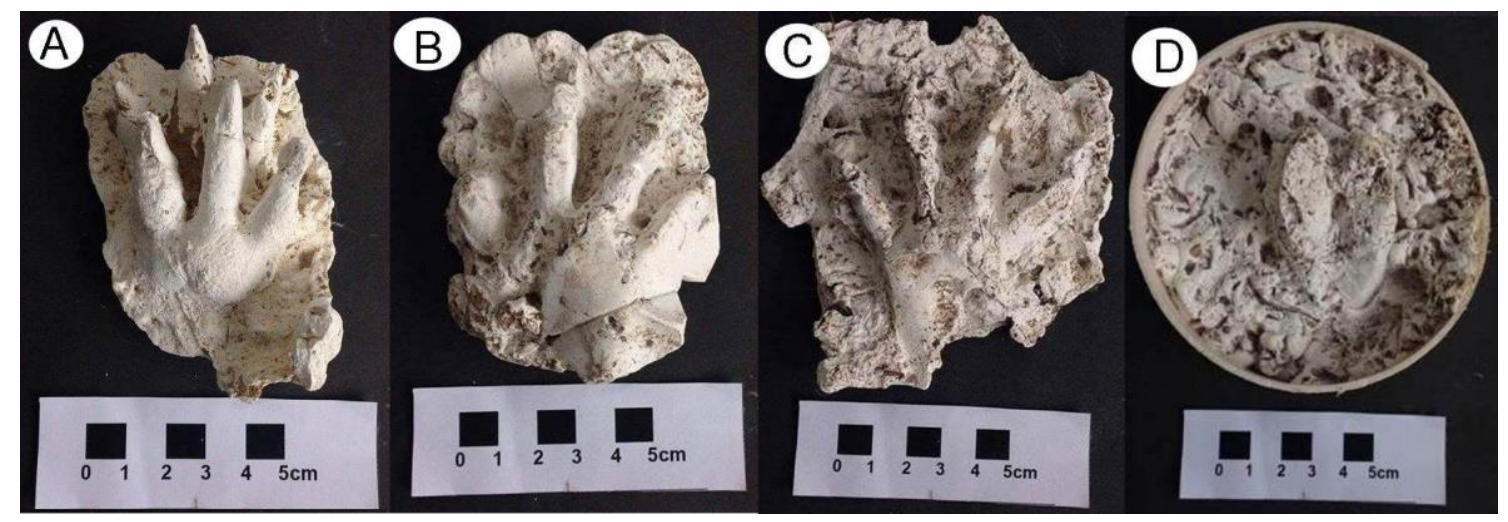

Figura 3: Pegadas de tatu-verdadeiro (Dasypus novemcinctus) (A), mão-pelada (Procyon cancrivorus) (B, mão, e C, pé) e caititú (Pecari tajacu) (D). 


\section{CONSIDERAÇÕES FINAIS}

A recuperação de vestígios e o uso de armadilhas fotográficas na Serra da Jiboia vêm se mostrando importantes para a obtenção de um inventário mais completo da mastofauna da região, mostrando de forma indireta (no caso dos vestígios) e direta (registros fotográficos) a presença de espécies que não aparecem através de outros métodos de amostragem, como a captura em armadilhas de atração por isca.

\section{REFERÊNCIAS}

ABREU JR., E.F. \& KÖHLER, A. 2009. Mammalian fauna of medium and large sized in the RPPN of UNISC, RS, Brazil. Biota Neotropica, vol. 9, no. 4.

BORGES, P.A.L. \& TOMÁS, W.M. 2004. Guia de rastros e outros vestígios de mamíferos do Pantanal. Embrapa Pantanal, Corumbá, 148 p.

EMMONS, L. \& FEER, F. 1997. Neotropical rainforest mammals. A field guide. Second edition. Chicago: The University of Chicago Press 307p.

GLEN, A.S.; COCKBURN, S.; NICHOLS, M.; EKANAYAKE, J. \& WARBURTON, B. 2013. Optimising camera traps for monitoring small mammals. PlosOne, 8:1-7.

MAZZOLLI, M. 2006. Persistência e riqueza de mamíferos focais em sistemas agropecuários no planalto meridional brasileiro. Tese de Doutorado, Universidade Federal do Rio Grande do Sul, Porto Alegre.

O'CONNELL; A.F., NICHOLS; J.D. \& KARANTH, K.U. 2011. Camera traps in animal ecology: methods and analyses. New York: Springer, 271p.

REIS, N.R.; PERACHI, A. L.; ROSSANEIS, B.K. \& FREGONEZI, M.N. 2010. Técnicas de estudo aplicadas aos mamíferos silvestres brasileiros. Rio de Janeiro: Technical Books, $275 \mathrm{p}$.

REIS, N.R.; PERACHI, A.L.; PEDRO, W.A. \& LIMA, I.P. 2011. Mamíferos do Brasil. 2ed. Londrina, 439p.

SILVEIRA, L.; JÁCOMO, A.T.A. \& DINIZ-FILHO, J.A.F. 2003. Camera trap, line transect census and track surveys: a comparative evaluation. Biological Conservation, 114:351-355.

SINCLAIR, A. R. E. 2003. Mammal Population Regulation, Keystone Processes and Ecosystem Dynamics. Philosophical Transactions: Biological Sciences, 358(1438):17291740 .

TONHASCA JR., A. 2005. Ecologia e história natural da Mata Atlântica. Interciência, Rio de Janeiro, 197 p. 